\title{
Un poco de la Historia de la Mina de Dificultad
}

\author{
A bit of the History of the Difficulty Mine
}

José Ulises Rodríguez-Sánchez ${ }^{a}$

\begin{abstract}
:
When talking about Real del Monte, the first thing that comes to mind is: a beautiful, historical and cultural place, it certainly is.

But it is a town that still keeps many secrets, and many of those secrets are in the mines that exploited the Vizcaína Vein, the great vein that for 200 years was exploited and that now these mines are "Historical Patrimony of the Nation", without place they doubtlessly earned a place in the history of Mexico and the World, since the great Vizcaína vein is one of the largest and most profitable in the history of world mining.
\end{abstract}

Keywords:

Real del Monte, Vizvaína Vein, Historial National Heritage

\section{Resumen:}

Al hablar de Real del Monte, lo primero que nos viene a la mente es: un lugar hermoso, histórico y cultural, sin duda lo es.

Pero es un pueblo que aún guarda muchos secretos, y mucho de esos secretos están en las minas que explotaron la Veta Vizcaína, la gran veta que por 200 años fue explotada y que ahora dichas minas son "Patrimonio Histórico de la Nación", sin lugar a duda se ganaron un lugar en la historia de México y del Mundo, ya que la gran veta Vizcaína es una de las más grandes y más provechosas en la historia de la minería mundial.

\section{Palabras Clave:}

Real del Monte, Veta Vizcaína, Patrimonio Histórico de la Nación

El presente ensayo se basa en mi investigación personal llevada a cabo en Real del Monte, en la historia de dicho lugar. El objetivo del que parte es dar a conocer algunos de los antecedentes históricos de Real del Monte, ya que en dicho lugar ocurrieron sucesos históricos que marcaron el rumbo de la region, el estado de Hidalgo y del país.

Es muy importante conocer la historia de Real del Monte, ya que ocurrieron sucesos que tuvieron relevancia en la historia nacional e internacional. Antes de la conquista de México, Real del Monte era ocupado por personas de lengua Otomí, de ahí proviene el nombre de Magotsi, palabra otomí compuesta de ma que significa "altura" y gohtsi que significa "paso", dado que precisamente era el

\footnotetext{
${ }^{1}$ http://www.dimensionantropologica.inah.gob.mx/?p=1388 $\quad 2$ Íbidem.
}

paso que unía al señorío de Meztitlan con la gran Tenochtitlan. ${ }^{1}$

En tiempos del virreinato se daba el nombre real por estar bajo el dominio de la corona española, y del monte por estar rodeado de montes; por tal motivo se le nombró Real del Monte. ${ }^{2}$

Ubicada en el Real del Monte, jurisdicción de Pachuca, la gran veta Vizcaína se hizo en un lugar muy principal entre todos los minerales del reino por su riqueza, y por ella es conocido en todo el orbe el Real del Monte, el camino en donde la naturaleza la ubicó.

Hacia el siglo XVIII durante el virreinato, sobresalió entre todos, el señor don Pedro Romero de Terreros, caballero de la orden de Calatrava, primer conde de Regla, el 
vasallo más rico de la monarquía en su tiempo. La veta Vizcaína proporcionó a su mano liberal esos inmensos tesoros que religiosos y leal ofreció al pie del altar y el trono. (Castelazo, 1820).

Con el paso del tiempo don Pedro Romero de Terreros se hacía más poderoso, pero también surgían problemas entre los mineros que trabajaban en sus minas. Don Pedro Romero de Terreros decidió suprimir el partido y reducir los jornales de 4 a 3 reales por jornada laboral y aumentar las cargas de trabajo para obtener mayores ganancias.

Los jornales eran los pagos en dinero que se hacían a los mineros en asignación diaria y pago semanal, y el partido era un incentivo libre para los mineros, era un costal de mineral que obtenía el minero y que lo dividía al final de jornada entre él y el dueño. Debido a estos sucesos, en 1766 estalló una huelga de mineros, la cual fue considerada como la más importante en el México colonial y la primera de América.

Esta huelga causó grandes problemas para don Pedro Romero de Terreros, ya que afectó su gran reputación ganada durante años, además de pérdidas ocasionadas por la suspensión en la explotación de los minerales.

Finalmente, un grupo pequeño de operarios, había comenzado a reunirse clandestinamente para elaborar documentos que contenían las exigencias de los mineros, fue tal la presión que don Pedro Romero de Terreros cedió a las peticiones y se reanudó el trabajo en las minas.

A la muerte de don Pedro Romero de Terreros en 1781, su hijo José María Romero de Terreros heredo el poder sobre la veta Vizcaína, sin embargo, no tenía el potencial de su padre y mucho menos el sustento económico. José María Romero de Terreros dirigió su atención hacia Inglaterra, solicitó que Josef Rodrigo Castelazo escribiera un opúsculo, sobre las condiciones en que se encontraban las minas. Para 1823 José María Romero de Terreros envió a Londres una propuesta de inversión, se dirigió a John Taylor, empresario minero de Cornwall. La respuesta fue favorable, para el 16 de enero de 1824 se da el alquiler de las minas en Real del Monte. El 4 de febrero de 1824 hubo una segunda reunión entre José María Romero de Terreros, John Taylor y su socio Tomas Murphy, en la reunión se crea la Compañía de Caballeros Aventureros de las Minas de Pachuca y Real del Monte. ${ }^{3}$ El primer grupo compuesto por algunos técnicos ingleses, comandado por el técnico James Vetch arribó a México el 11 de junio de 1824 y se establecieron en el distrito minero de Real del Monte y Pachuca para realizar el reconocimiento de las minas. Finalmente, los principales lugares de trabajo de las minas en Real del Monte fueron la mina del tiro de Dificultad y la mina del tiro de Acosta. Con la llegada de los ingenieros y mineros de origen británico y aleman, el resultado fue una mezcla se hábitos culturales y sociales, pues con el paso de los años los pobladores de Real del Monte y Pachuca adoptaron formas de vestir, costumbres, comida típica y el futbol, que en la actualidad es el deporte nacional.

Después de que la compañía tomó posesión en 1824, los ingenieros ingleses reportaron que la mayor parte de los tiros verticales que comunicaban con los labrados subterráneos estaban quebrados y hundidos, y que solo podían reconocerse por los enormes hundidos y terreros que se habían formado cerca de ellos, Real del Monte estaba muy abatido y la población muy disminuida.

Para emprender el desagüe de estas profundas minas, consistía en la superioridad del uso de las máquinas de vapor y los fletes de bombas sobre el costoso desagüe mexicano por medio de botas de cuero levantadas por malacates de caballos, se enviaron de Inglaterra a México cierto número de maquinistas y operarios de mina, con cinco máquinas de vapor de desagüe, un mortero de vapor y dos pequeñas máquinas de aserrar, todo lo cual formaba el cargo de tres buques de 300 toneladas de porte cada uno y fue desembarcado en el mes de Mayo de 1825. (Burkart, 1969)

La llegada de maquinaria impulsada por vapor traída por los ingleses, fue una gran ventaja para la explotación de las minas en Real del monte, debido a esto el proceso de explotación de minas fue acelerado gracias a la nueva tecnología. Esta es una parte importante ya que cambió la forma de explotar las minas y de adquirir los minerales que se hallaban en la veta Vizcaína.

En 1885 la casa Paschker und Kaestner en Freiberg, construyó una máquina de columna de agua para el desagüe de la mina Morán; y después de un estudio realizado por los directivos de la Compañía Real del Monte y Pachuca, que incluyó la visita a fábricas alemanas, se optó por adquirir una máquina para la mina Dificultad en Chemnitz, Alemania que, si bien resultaba más costosa a una oferta que se tenía de Cornwall, se justificaba por la economía que se tendría en el consumo del combustible.

Éste estaba compuesto por una bomba de sistema Rittinger con potencia de 900 caballos de vapor, una bomba perforadora para profundizar el tiro hasta 485 metros, y un aparato Eschwingel completo para subir y bajar la bomba. Una serie de refacciones para ambas bombas, además de tubería, tornillaje, empalmes de goma y llaves de tuercas, se hicieron necesarios dos neutralizadores o balanzones hidráulicos que sirvieron

${ }^{3}$ http://migracioncornica.34.dot.unam/392524.com 
para contrabalancear las 85 toneladas de peso de las cadenas de transmisión. En octubre de 1888 el montador ruso Anatolio Mertzenfeld informó que podía terminar el trabajo de acondicionamiento del tiro, con personal de la Maestranza de Real del Monte sin que fuera necesario contratar más personal en Alemania. (Ordoñez y Rangel, 1889).

El equipo tendría un peso total de 310 toneladas y un costo de $\$ 130950$ marcos alemanes. Se estableció un contrato de personal; entre septiembre de 1888 y febrero de 1890, llegaron a Real del Monte montadores, carpinteros, ingenieros, bomberos, albañiles, ademadores y maquinistas desde Alemania. La casa de máquinas edificada resultó ser un edificio de dimensiones nunca vistas en la región y el país: $14 \mathrm{~m}$ de ancho, $26 \mathrm{~m}$ de largo y $20 \mathrm{~m}$ de alto, techo de armaduras de fierro cubiertas de lámina acanalada, y tres cuerpos de ventanas.

En el interior de ella una poderosa máquina del sistema Woolf de doble efecto, con bombas Rittinger, un contrapeso hidráulico y un malacate fijo de 80 caballos. A un costado una chimenea de $50 \mathrm{~m}$ de altura y forma octagonal, también única en la zona.

Cabe señalar que esta chimenea se puede observar casi desde cualquier punto de Real del Monte.

Fue la máquina para desaguar minas más grande y más potente instalada en México y Latinoamérica. (Crespo, 1901)

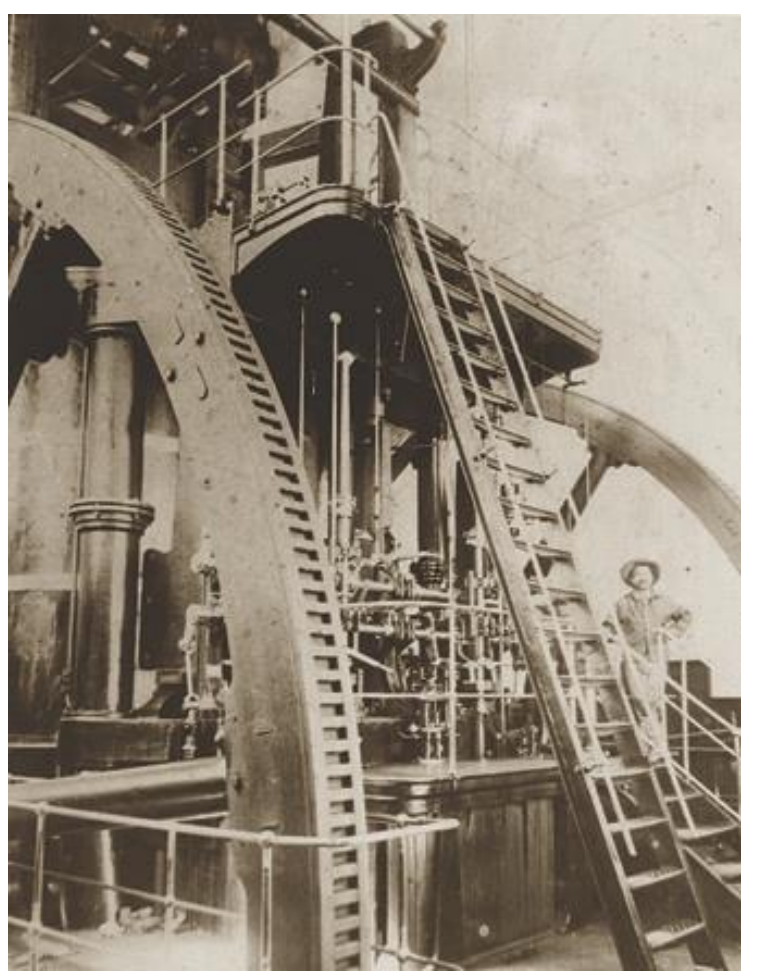

Máquina de vapor, 1890 Plata/gelatina.

Fototeca AHMM, A.C.
La instalación, inauguración y puesta en funcionamiento de la máquina de vapor de la mina La Dificultad en Real del Monte, tuvo un impacto que aún perdura en el paisaje, en la economía, en las relaciones laborales, en la producción metalífera, en la vida misma de un pueblo. (García, 1890)

Quizá de esto fueron conscientes quienes trabajaron para hacerlo posible y posaron en las escaleras de acceso a la casa de máquinas, para una fotografía que sería histórica y que es una muestra de que al final de la historia, trabajadores extranjeros y mexicanos aprendieron a convivir, a intercambiar experiencias y conocimientos, a superar dificultades en pos de la búsqueda del preciado metal.

La máquina alemana de La Dificultad hizo posible abrazar un sueño anhelado desde la época virreinal: desaguar las minas de la zona; y al poco tiempo concluir una etapa histórica y tecnológica: la era del vapor en Real del Monte.

El desagüe realizado por la máquina de La Dificultad, cuyo costo total incluido su traslado desde Alemania a Real del Monte, la habilitación del sitio y su instalación fue de \$423,036 francos alemanes, permitió continuar los trabajos de exploración y explotación de la veta Vizcaína y dar el salto al nuevo siglo; dio tranquilidad y ganancias a los accionistas de la empresa, de \$153 319 Libras en 1889 a \$ 529018 Libras en 1891. A los trabajadores les otorgó la seguridad de mantener su fuente de ingresos, y a la población tranquilidad porque sabía que su vida dependía, como en todos los pueblos mineros, de la continuidad productiva; de ahí la gran fiesta que organizaron con motivo de la puesta en marcha de la máquina. (AH CRDMYP, 1888).

Como consecuencias de la acelerada explotación de las minas, gracias a la llegada de la nueva tecnología, los mineros mexicanos y extranjeros desarrollaron muchas y muy variadas enfermedades, pero lamentablemente no se cuentan con registros que nos permitan conocer los grados de incidencias o las tasas de mortandad que suponemos muy altas.

Las enfermedades más comunes eran la silicosis, es la más común y la más dolorosa, es una enfermedad pulmonar de origen ambiental producida por la inhalación de partículas de sílice libre o cuarzo cristalizado. La fibrosis pulmonar progresiva que se produce suele depender de la dosis que se inhala o inspire y puede ocurrir años después de su exposición. Entre otras esta la Blefaraconiosis, anquilostomiasis y la leptospirosis. Además de eso, la deforestación en el lugar no tardó en hacerse visible, ya que el malacate funcionaba con el vapor producido de 4 calderas, cada una consumía un total de 20000 litros de agua y 1 tonelada de leña al día. 
En La Dificultad se instaló en 1938 la central eléctrica que abasteció de esta energía a las minas de la Compañía de Real del Monte y Pachuca, dando fin a un siglo en cual se hizo efectiva la utilidad de las máquinas de vapor.

\section{Bibliografía}

AH CRDMYP, Archivo Histórico de la Compañía de Real del Monte y Pachuca, 1888: Fondo del Siglo XIX. Bitácora de la Administración de Minas de Real del Monte.

Burkart, J. (1969) 1989: Memoria sobre la Explotación de Minas de los Distritos de Pachuca y Real del Monte de México, Trad. Y Notas Miguel Velázquez de León, Ed. Universidad Autónoma del Estado de Hidalgo, Pachuca, México.

Castelazo, J. R. de. 1820: Manifiesto de la Riqueza de la Negociación de Minas conocida por la veta Vizcaína, México, impreso en la Casa de Ontiveros, Méjico..

Crespo y Martínez, G. 1901: La Evolución Minera en México, su evolución social, Ed. Independiente, México.

García Moreno, J. (16 de enero 1890) "Libertad para todo y para todos, menos para el mal y para los malvados", El Heraldo. Diario católico, México, núm. 256, año 1.

Ordoñez, E. y Rangel, M.1899: El Real del Monte, Boletín del Instituto Geológico de México, Oficina Tipográfica de la Secretaría de Fomento.

http://www.dimensionantropologica.inah.gob.mx/?p=1388 Consultado 20/02/ 2018

http://migracioncornica.34.dot.unam/392524.com Consultado: 28/02/18 